\title{
A review of economic evaluations of genetic testing services and interventions (2004-2009)
}

\author{
Sandjar Djalalov, PhD ${ }^{1,2}$, Zahra Musa, MHA ${ }^{2}$, Michael Mendelson, MA ${ }^{3}$, Katherine Siminovitch, MD ${ }^{4,5}$, \\ and Jeffrey Hoch, PhD $D^{1,2,4}$
}

\begin{abstract}
To review economic evaluations of genetic interventions published between 2004 and 2009 and assess the quality of the identified studies, we searched for economic evaluations of genetic testing and interventions published between 2004 and 2009. Studies that met inclusion criteria were reviewed and their quality then assessed using a validated instrument - the Quality of Health Economic Studies. Of 54 articles identified, 26 met study criteria and were included in the review. These studies span a number of clinical indications, genetic tests, and interventions, but the majority ( $92 \%$ ) focuses on genetic interventions for preventive screening and increasing treatment efficacy. The mean quality score for the reviewed studies was 89.8. Comparison of the quality of different study types revealed that cost-utility studies and studies that used a combined decision tree and Markov model had the highest mean quality scores. Clear statements regarding bias, funding source, and study perspective were commonly lacking in the reviewed studies. Although the reviewed studies were of fairly high quality, we found Quality of Health Economic Studies methodology for grading the quality of economic evaluations challenging and observed no statistically significant improvement in quality of studies between the periods of 1995-2004 and 2004-2009. Genet Med 2011:13(2):89-94.
\end{abstract}

Key Words: economic evaluation, genetic testing, quality assessment, cost-effectiveness analysis, personalized medicine

G enetic testing has the potential to benefit the population in many ways, including enabling early detection of patients with hereditary predispositions to specific diseases, individualization of drug therapy, and development of gene-based drug therapies. ${ }^{1}$ However, this is not without controversy. For example, some claim genetic testing has had little impact on current clinical practice, a situation reflecting ethical, financial, medical, scientific, and/or sociologic impediments to genetic screening and service programs. ${ }^{2}$ One such scientific impediment includes the lack of validation studies on the associations between genetic testing and outcomes. Clarifying the economic implications associated with genetic testing provides additional information for decision makers with scarce resources.

In an environment of limited health care resources, costs and the clinical effectiveness of medical interventions are factors

From the ${ }^{1}$ Centre for Research on Inner City Health, Keenan Research Centre, Li Ka Shing Knowledge Institute, St. Michael's Hospital; ${ }^{2}$ Pharmacoeconomics Research Unit, Cancer Care Ontario; ${ }^{3}$ Caledon Institute of Social Policy; ${ }^{4}$ Faculty of Medicine, University of Toronto; and ${ }^{5}$ Samuel Lunenfeld Research Institute, Mount Sinai Hospital, Toronto, Ontario, Canada. Jeffrey Hoch, PhD, Pharmacoeconomics Research Unit, Cancer Care Ontario, 620 University Ave, Toronto, ON, Canada M5G 2L7. E-mail: jeffrey.hoch@utoronto.ca.

Disclosure: See Acknowledgements on final page.

Submitted for publication August 19, 2010.

Accepted for publication October 5, 2010.

Published online ahead of print January 5, 2011.

DOI: $10.1097 /$ GIM.0b013e3182003294 considered in funding decisions. Economic evaluation provides a potentially valuable tool to prioritize health interventions, allowing identification of interventions that maximize health gains per dollar. Economic evaluation is defined as "the comparative analysis of alternative courses of action in terms of both their costs and consequences." 3 Cost-effectiveness analysis (CEA), a type of economic evaluation, provides a quantitative framework for evaluating interventions or treatments and is used by decision makers to evaluate new health technologies. As a result, there is a corresponding increase in the number of economic evaluations published and an increased demand for systematic reviews of these studies. This is particularly true of genetic testing and services, an area now undergoing an unprecedented pace of new discovery with potential clinical application. ${ }^{4}$

By reviewing economic evaluations of genetic testing services published between January 1990 and August 2004, Carlson et al. ${ }^{5}$ provided meaningful insight into the quality and interpretation of such studies. Since 2004, there have been a considerable number of economic evaluations published on genetic testing and interventions; however, there have been few published reviews on the health economic evidence pertaining to these types of investments. Moreover, these reviews focused primarily on pharmacogenetic strategies in cancer, ${ }^{6,7}$ cystic fibrosis, ${ }^{8}$ and screening, ${ }^{2,9}$ and none assessed the quality of the economic evaluations.

Quality assessment of economic evaluations of new medical interventions is important to the health care community and in particular to decision makers because it may indicate which studies should be included when making evidence-informed policy or decisions pertaining to funding. For these reasons, we assessed more recent contributions in this area and accordingly undertook a review and quality assessment of economic evaluations of genetic testing and interventions published from 2004 to 2009 .

\section{METHODS}

In July 2009, we performed a literature search for economic evaluations on genetic testing and interventions published between September 2004 and June 2009. We used the same search terms and databases as Carlson et al., "5 wich included terms as follows: "cost-effectiveness," "cost-benefit," "cost-minimization," "cost-utility," "economics," "gene therapy," "pharmacogenetics," "genetic screening," and "genotype" and used databases such as PubMed, Medline, Proquest, LexisNexis, Expanded Academic Index, The Harvard Review of Economic Analyses (http://www.hsph.harvard.edu/cearegistry/), PsycINFO, National Institute for Clinical Excellence (http://www.nice.org.uk), and the Canadian Agency for Drugs and Technologies in Health (http://www.cadth.ca/index.php/en/home).

For inclusion in our review, studies had to meet the following criteria: (1) a focus on detecting heritable disease, (2) reporting on an economic evaluation of a genetic test or service, and (3) publication in a peer-reviewed journal. Studies that were not published in English, full-sized articles, or related to interven- 
tions intended for humans were excluded. Studies on the genotyping of viral genomes such as hepatitis or human immunodeficiency virus were also excluded.

All studies meeting inclusion criteria were reviewed and information on design and model characteristics, incremental cost-effectiveness ratios (ICERs), and conclusions then extracted. Summary statistics were calculated for the type of intervention, economic analysis, model, perspective, and sensitivity analysis. Conclusions for studies in similar clinical areas were summarized. ICERs extracted in this review and those cited by Carlson et al. ${ }^{5}$ were graphed to observe trends through time. Each article identified through the literature review was also assessed for quality by two reviewers (S.D. and Z.M.) using the Quality of Health Economic Studies (QHES) instrument developed by Chiou et al. ${ }^{10}$ This grading system has 16 criteria, each of which is associated with a specific value, and the total quality scores can range from 0 to 100 . Before grading, both reviewers reached consensus on the interpretation of the criteria. Articles were then graded individually by each reviewer, with points deducted from the maximum score (100) for each criterion not addressed. After the independent reviews, the results were discussed, and either a consensus was reached for each score or a third reviewer (J.H.) was consulted to achieve a consensus score.

Once scores were obtained using the QHES grading system, the overall mean and subgroup means were calculated and compared with those reported by Carlson et al. ${ }^{5}$ The difference between mean scores was assessed for statistical significance using a one sample $t$ test. Because the magnitude of the quality score is based on the deduction of points for missed criteria, the number of studies that failed to address each quality criterion was tracked to identify major contributors to poor quality scores in the reviewed studies.

\section{RESULTS}

\section{Descriptive statistics of included studies}

The search strategy identified 631 publications. Among these, 54 satisfied the inclusion criteria based on their titles and abstracts. However, more complete review of the 54 full-text articles revealed only 26 to be economic evaluations, and, accordingly, all subsequent analyses focused on these 26 studies. ${ }^{1-36}$

The majority of these 26 studies were focused on genetic interventions for preventive screening $(46 \%)$ or increasing treatment efficacy (46\%). Most of the preventive screening interventions were for detecting specific genetic mutations in carriers with high risk of developing the associated disease. Genetic interventions aimed at increasing treatment efficacy mostly incorporated a pharmacogenomics approach. Of the only two studies $(8 \%)$ addressing other types of genetic interventions, one focused on prenatal screening to detect fragile $\mathrm{X}$ syndrome and the other on a diagnostic test for children with learning and developmental disabilities.

Table 1 presents descriptive statistics by type of economic analysis, model, perspective, and sensitivity analysis. All the studies reviewed used one of four types of economic analysis: CEA, cost-utility analysis (CUA), cost-benefit analysis (CBA), and costconsequence analysis (CCA). CEA, which calculates cost per single unit of effect or outcome, was the most common method $(65 \%)$, and cost-utility, which uses quality-adjusted life year, was the least common (12\%) method used across these studies.

Economic evaluations often use a model, such as a decision tree-which calculates "payoffs" of various possible choices represented as branches. Also Markov models are frequently used, which use a series of "states" that represent the disease
Table 1 Descriptive statistics and quality scores

\begin{tabular}{ccccc}
\hline & & $\begin{array}{c}\text { Mean } \\
\text { quality } \\
\text { score }\end{array}$ & $\begin{array}{c}\text { Range of } \\
\text { quality } \\
\text { scores }\end{array}$ \\
\hline
\end{tabular}

Type of economic analysis

\begin{tabular}{|c|c|c|c|c|}
\hline Cost-effectiveness & 17 & 65 & 89.5 & $65-100$ \\
\hline Cost-utility & 6 & 23 & 96.8 & $93-100$ \\
\hline $\begin{array}{l}\text { Cost-benefit and Cost- } \\
\text { consequence analyses }\end{array}$ & 3 & 12 & 54.0 & $41-71$ \\
\hline
\end{tabular}

Type of model

$\begin{array}{lrrrr}\text { Decision tree } & 12 & 46 & 89.4 & 65-100 \\ \begin{array}{l}\text { Decision tree and } \\ \text { Markov model }\end{array} & 8 & 31 & 94.5 & 84-100 \\ \text { Others } & 6 & 23 & 68.8 & 41-94\end{array}$

Type of sensitivity analysis

$\begin{array}{lrrrr}\text { One way } & 14 & 54 & 92.1 & 71-100 \\ \text { One way and two ways } & 3 & 12 & 91.3 & 77-100 \\ \text { Probabilistic } & 4 & 15 & 94.5 & 94-100 \\ \text { Not applicable } & 5 & 19 & 64.2 & 41-94\end{array}$

Perspective

\begin{tabular}{lrrrl} 
Healthcare & 13 & 50 & 88.7 & $49-100$ \\
Societal & 7 & 27 & 91.3 & $71-100$ \\
Not stated & 6 & 13 & 78.4 & $41-96$ \\
Overall & 26 & 100 & 89.8 & $41-100$ \\
\hline
\end{tabular}

process and are associated with a benefit and cost. ${ }^{3}$ Decision tree analysis was the most commonly used in the 26 reviewed studies (46\%). These models may also incorporate different study perspectives depending on the objectives of the study. Although the societal perspective, which includes all costs borne by the health system, patient, and society, is highly recommended by many guidelines, ${ }^{37-39}$ the studies reviewed here most frequently used the health care system perspective $(50 \%)$ with relatively few studies using a societal perspective (27\%).

Approaches to sensitivity analysis were also explored. Such analyses provide a means to characterize uncertainty in the model. The most simple sensitivity analysis is a one-way sensitivity analysis, where each model input is varied individually to investigate its impact. Two-way or multiway sensitivity analyses are extensions of a one-way sensitivity analysis. ${ }^{3}$ More than half of the studies included in this review used a one-way or two-way sensitivity analysis. Economic evaluation guidelines recommend the use of probabilistic sensitivity analysis (PSA), ${ }^{37}$ wherein a probability distribution for each model input is defined and simulations are run with the value of the model input being randomly drawn from the defined distributions. ${ }^{37}$ PSA was performed in $15 \%$ of the economic evaluations that were reviewed. Of the studies that did not conduct any sensitivity analysis, three were CBAs or CCAs and one used a modular simulation program to simulate all possible screening scenarios.

Sensitivity analysis also highlights which parameters have the largest impact on study results. The parameters that were 
Table 2 Overview of economic evaluations for genetic testing and interventions (2004-2009)

\begin{tabular}{|c|c|c|c|c|}
\hline Authors & Clinical condition & Intervention & $\begin{array}{l}\text { Type of } \\
\text { analysis }\end{array}$ & Result \\
\hline Winter et al. ${ }^{19}$ & Inflammatory bowel disease & Treatment efficacy & CEA & $£ 347 / \mathrm{LYG}$ \\
\hline Balmana et al. ${ }^{24}$ & Breast cancer & Preventive & CEA & $€ 4,294 / \mathrm{LYG}$ \\
\hline Phillips et al. ${ }^{25}$ & Familial long QT syndrome & Preventive & CEA & US\$2,500/LYG \\
\hline Kievit et al. ${ }^{26}$ & Colorectal cancer & Preventive & CEA & $€ 3,801 / \mathrm{LYG}$ \\
\hline Musci and Caughey ${ }^{11}$ & Fragile $\mathrm{X}$ syndrome & Preventive & CUA & US\$14,858/QALY \\
\hline Perlis et al. ${ }^{27}$ & Schizophrenia & Treatment efficacy & CUA & US\$47,705/QALY \\
\hline Dubinsky et al. ${ }^{20}$ & Crohn's disease & Treatment efficacy & CEA & $\begin{array}{l}\text { Dominant-earlier time to response and less } \\
\text { costly }\end{array}$ \\
\hline Warren et al..$^{15}$ & Cystic fibrosis & Preventive & CEA & AUD $\$ 5,834 /$ additional carrier detected \\
\hline Hollingsworth and Harris ${ }^{34}$ & Fragile $\mathrm{X}$ syndrome & Other & CBA & Cost-neutral \\
\hline Breheny et al. ${ }^{28}$ & Colorectal cancer & Preventive & CEA & $\begin{array}{l}\text { AUD } \$ 14,783-15,460 / \text { colorectal cancer-free } \\
\text { year }\end{array}$ \\
\hline Meckley and Veenstra. ${ }^{12}$ & Hypertension & Treatment efficacy & CUA & Dominant-more QALYs and less costly \\
\hline Priest et al. ${ }^{21}$ & Inflammatory bowel disease & Treatment efficacy & CEA & NZ\$11,000 cost savings \\
\hline Morelle et al. ${ }^{29}$ & Breast cancer & Treatment efficacy & CEA & $\begin{array}{l}\text { No strategy was cost-effective under criteria } \\
\text { specified }\end{array}$ \\
\hline Costa-Scharplatz et al. ${ }^{13}$ & Nephropathy & Treatment efficacy & CEA & $\begin{array}{l}€ 365,000 \text { net cost saved } / 1,000 \text { years and } 9 \\
\text { LYG free of end stage renal disease }\end{array}$ \\
\hline Olsen et al. ${ }^{30}$ & Colorectal cancer & Preventive & CEA & $€ 980-1,947 / \mathrm{LYG}$ \\
\hline Neilsen et al. ${ }^{31}$ & Colorectal cancer & Preventive & CUA & $€ 25,000-51,500 / \mathrm{QALY}$ \\
\hline Veenstra et al. ${ }^{14}$ & Cystic fibrosis & Treatment efficacy & CUA & US\$79,300/QALY \\
\hline Gagné et al. ${ }^{17}$ & Hereditary hemochromatosis & Preventive & CEA & $\begin{array}{l}\text { Dominant-additional life expectancy and } \\
\text { less costly }\end{array}$ \\
\hline Newman et al. ${ }^{35}$ & Learning disability & Preventive & $\mathrm{CCA}$ & Cost per positive case varies \\
\hline Wei et al. ${ }^{16}$ & Cystic fibrosis & Preventive & CBA & Cost saving \\
\hline Welton et al. ${ }^{22}$ & Smoking cessation & Treatment efficacy & CUA & Dominant-highest incremental net benefit \\
\hline Heitjan et al..$^{23}$ & Smoking cessation & Treatment efficacy & CEA & US\$2,985/LYG \\
\hline Mersereau et al. ${ }^{32}$ & Prevention of aneuploid birth & Preventive & CEA & US\$50,687/healthy infant \\
\hline Cooper et al. ${ }^{18}$ & $\begin{array}{l}\text { Hereditary } \\
\text { haemochromatosis }\end{array}$ & Treatment efficacy & CEA & $£ 123 /$ case detected \\
\hline Eckman et al. ${ }^{33}$ & Nonvalvular atrial fibrillation & Treatment efficacy & CEA & US\$170,000/QALY \\
\hline Rogowski $^{8}$ & Hereditary hemochromatosis & Preventive & CEA & $€ 41,000-161,000 / \mathrm{LYG}$ \\
\hline
\end{tabular}

CEA, cost-effectiveness analysis; CUA, cost-utility analysis; CBA, cost-benefit analysis; CCA, cost-consequence analysis; QALY, quality-adjusted life year; LYG, life-years gained.

chosen to be varied in the sensitivity analyses were different in each of the studies reviewed. Approximately $60 \%$ of studies varied the cost of genetic testing, but only a few included the specificity and sensitivity of the genetic test in their sensitivity analysis. Although prevalence of genotype distribution among a population can have a significant impact on CEA results, this factor was considered in only $25 \%$ of the studies.

\section{Overview of included studies}

The studies included in this review span a number of clinical indications, genetic tests and interventions that are summarized in Table 2. Conclusions pertaining to cost-effectiveness of the genetic testing and interventions are listed according to clinical area. As summarized in the table, four studies evaluated the cost-effectiveness of colorectal cancer screening or surveillance of high-risk individuals, ${ }^{26,28,30,31}$ and, within the specified scenarios, genetic testing and screening of high-risk individuals were considered to be cost-effective.

The cost-effectiveness of prenatal screening for cystic fibrosis was also examined in two studies. ${ }^{15,16}$ Of these, one study found a school-based screening program for genetic carriers of cystic fibrosis to be of comparable cost-effectiveness with other 


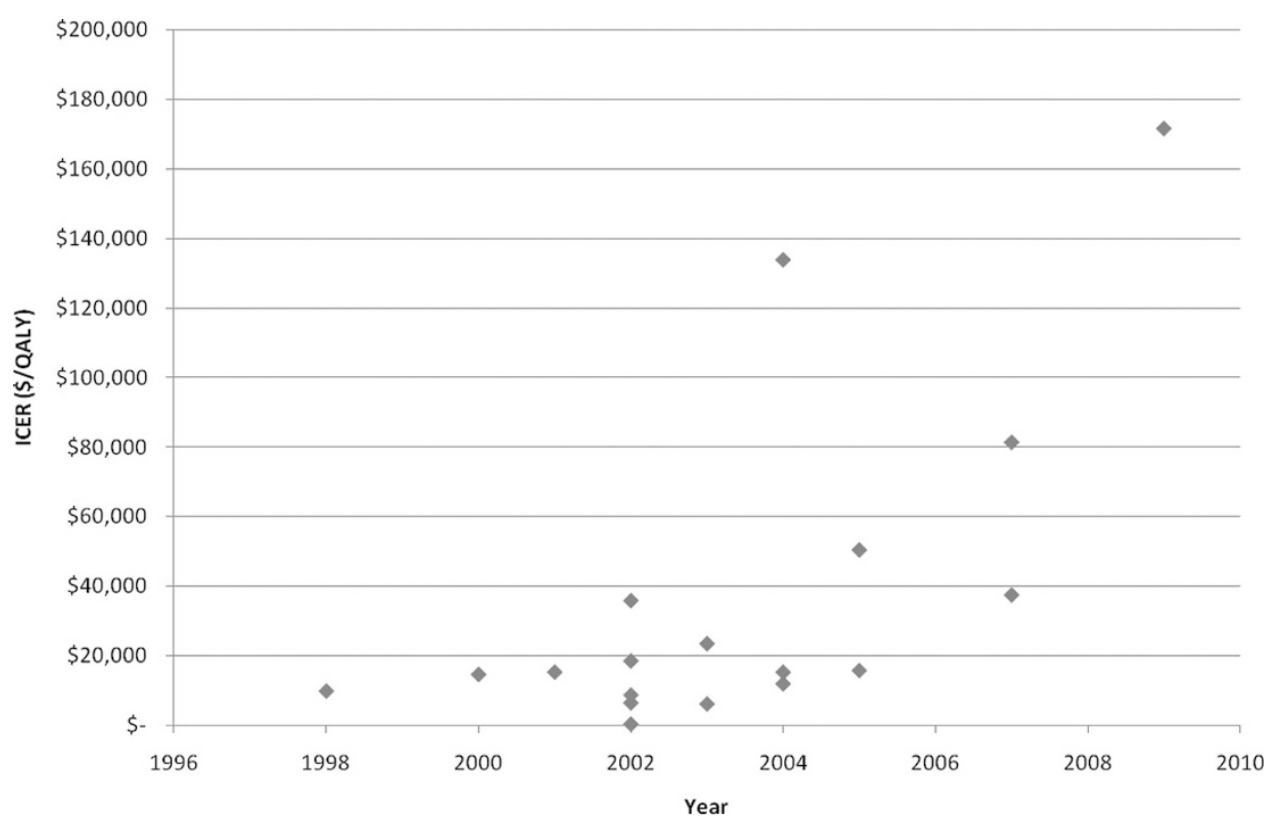

Fig. 1. Trends in ICERs (\$/QALY) for genetic testing interventions (1998-2009). QALY, quality-adjusted life year.

prenatal cystic fibrosis screening programs. ${ }^{15}$ The other study concluded that screening for cystic fibrosis resulted in costsavings compared with nonscreening; in other words, the cost of screening was less than the lifetime costs incurred by patients with cystic fibrosis. ${ }^{16}$

Two other studies performed economic evaluations of different population screening scenarios for hereditary hemochromatosis. ${ }^{17,36}$ Both studies not only concluded that certain scenarios of population-based screening can be cost-effective but also noted considerable uncertainty in interpreting the data. One study, for example, cited discounting of outcomes and adherence of patients to preventative phlebotomy as influential parameters. ${ }^{36}$ The other study concluded that cost-effectiveness was dependent on the mutation penetrance and prevalence in a population. ${ }^{17}$

Three more studies evaluated the cost-effectiveness of genetic testing for thiopurine methyltransferase variants in patients with inflammatory bowel disease. ${ }^{19-21}$ Among these studies, one concluded that pretreatment genetic screening in inflammatory bowel disease patients represents good value for money with cost-effectiveness estimates that compare favorably to other health interventions. ${ }^{19}$ In a second study, it was suggested that phenotype testing alone results in cost-savings compared with genotype testing. ${ }^{21}$ The third study compared genetic testing, metabolite monitoring, and usual care and found that genetic testing and metabolite monitoring dominate usual care. $^{20}$

The cost-effectiveness of pharmacogenetic testing for smoking cessation treatment was evaluated by two other of the studies reviewed. ${ }^{22,23}$ Both studies included nicotine replacement and bupropion pharmacotherapy among the strategies evaluated. Results of one study indicated the use of genetic testing in tailoring smoking cessation treatment to be costeffective under certain assumptions. ${ }^{23}$ By contrast, the other study cautioned against assuming that genetic tailoring of treatment would be more cost-effective than current models for pharmacotherapy. ${ }^{22}$

Six studies included in this review were CUAs, but only five of these stated ICERs. The ICERs identified in this review
(2004-2009) and those from the review by Carlson et al. ${ }^{5}$ (1997-2004) were plotted to identify any trends over time (Fig. 1). The trend appears to be in an upward direction.

\section{Quality of included studies}

A quality score was assigned for each article by each reviewer using the QHES method. ${ }^{10}$ The correlation between the two reviewers' scores is 0.77 . The mean quality score for the reviewed studies was 89.8 with a range of $41-100$. The mean quality score and range were also calculated by subgroups: type of economic analysis, model, sensitivity analysis, and perspective (Table 1). Cost-utility studies had the highest mean quality score of 96.8, and, when compared with studies that used other types of economic analysis, cost analyses received the lowest mean quality score within this subgroup. Studies using a combined decision tree and Markov model received a higher mean quality score of 94.5 relative to studies that used only a decision tree or a simulation model. Studies that did not include a sensitivity analysis received a lower mean quality score than those that did.

Although the mean quality score from this review (89.77) was 2.72 points higher than the mean quality score from the review by Carlson et al. ${ }^{5}(87.05)$, this difference is not statistically significant $(P$ value $<0.35)$

The number of studies in this review that did not address specific quality criteria were also calculated (Table 3). This analysis revealed that the most frequently missed criteria was a discussion of bias (53.9\%) disclosure of funding source (30.8\%), and statement of study perspective (19.2\%).

\section{DISCUSSION}

The studies included in this review of economic evaluations for genetic testing and interventions were all published between 2004 and 2009, spanned a number of clinical indications, and used a variety of modeling techniques and assumptions. A few reviews of economic evaluation of genetic tests have been published since 2004, but the focus in these articles has been on 
Table 3 Missing quality criteria in reviewed studies

\begin{tabular}{lcc}
\hline No. & \\
Criteria (points) & studies & Percentage \\
\hline
\end{tabular}

Did the author(s) explicitly discuss direction

14

53.9

and magnitude of potential biases? (6)

Was there a statement disclosing the source of funding for the study? (3)

Were the perspective of the analysis (societal, third-party payer, etc.) and reasons for its selection stated? (4)

Did the analytic horizon allow time for all relevant and important outcomes? Were benefits and costs that went beyond 1 year discounted (3-5\%) and justification given for the discount rate? (7)

Were the economic model (including structure), study methods and analysis, and the components of the numerator and denominator displayed in a clear transparent manner? (8)

Were the health outcomes measures/scales valid and reliable? If previously tested valid and reliable measures were not available, was justification given for the measures/scales used? (7)

Were the choice of economic model, main assumptions, and limitations of the study stated and justified? (7)

Was uncertainty handled by (1) statistical analysis to address random events and (2) sensitivity analysis to cover a range of assumptions? (9)

Were variable estimates used in the analysis from the best available source (i.e., randomized control trial-best, expert opinion-Worst)? (8)

Was the study objective presented in a clear, specific, and measurable manner? (7)

Was the measurement of costs appropriate and the methodology for the estimation of quantities and unit costs clearly described? (8)

Was incremental analysis performed between alternatives for resources and costs? (6)

Was the methodology for data abstraction (including value health states and other benefits) stated? (5)

Were the primary outcome measure(s) for the economic evaluation clearly stated and were the major short-term, long-term, and negative outcomes included? (6)

Were the conclusions/recommendations of the study justified and based on the study results? (8)

If estimates came from a subgroup analysis, were the groups prespecified at the beginning of the study? (1) pharmacogenetic strategies. ${ }^{2,4-9}$ One review by Vegter et al., ${ }^{9}$ for example, examined the adherence of pharmacogenetic screening economic analyses to current guidelines. This study considered four aspects of pharmacoeconomic analysis when assessing the adherence to guidelines: (1) sensitivity analysis, (2) time horizon and discounting, (3) study perspective, and (4) review of evidence. The authors found that most economic evaluations of genetic interventions lacked standardization with respect to analysis perspective, sensitivity analysis specifics, and discount rates. ${ }^{9}$ In contrast, our review on the quality of economic evaluations of genetic services incorporated a more comprehensive quantitative methodology that includes not only the four aspects considered in the study by Vegter et al. ${ }^{9}$ but also 12 other quality criteria such as bias, assumptions, limitations, etc.

The analysis of subgroup quality scores revealed a number of interesting trends. Cost-benefit and cost-consequence studies, for example, had the lowest mean quality score, when compared with CEA and CUA. This finding may reflect the fact that many QHES criteria pertain to outcomes, and CBA and CCA consider outcomes differently. As is consistent with current recommendations by economics evaluation guidelines, the studies that performed PSA had higher quality scores, when compared with studies that used other types of sensitivity analysis. It is likely that authors who heeded guideline recommendations for sensitivity analysis also followed guidelines when constructing other parts of their evaluation, which would explain the observed trend between the use of PSA and higher quality scores.

Although there was a small difference between the overall mean quality score from the period 1990-2004 to the period 2004-2009, the difference was found to be statistically insignificant, implying little change in the quality of genetic intervention-focused economic evaluations between 1990-2004 and 2004-2009. One would have predicted an improvement in quality over this period in view of the increase in published guidelines for economic evaluation. The basis for the lack of such improvement is not clear but may reflect our study's higher proportion of CBA and CCA studies (which tend to have lower quality scores) or a ceiling effect.

The most commonly missed quality criterion in our reviewdiscussion of bias- differs from that most commonly missed in the review by Carlson et al. ${ }^{5}$ - statement of perspective. This discrepancy may be due to changes in journal policy, with a statement of perspective now being mandated by many journals.

Importantly, our study has a few limitations that need to be considered in terms of interpreting our findings. One such limitation relates to the terms genetics and genomics that are very broad and include a wide field of biomedical research that is not necessarily fully captured by our review. In addition, economic evaluations of genetic interventions tend to be improperly indexed and may be hidden in databases under diseasespecific terms. Circumventing this problem to ensure all studies pertaining to genetic interventions were captured would require a manual review of all economic evaluations in a plethora of disease fields. Another study limitation derives from the application of QHES grading methodology. We found some QHES criteria to be vague and difficult to apply. Some criteria are open to multiple interpretations, and many criteria include several considerations, which a single score may fail to capture. An example of such a criterion is "Did the analytic horizon allow time for all relevant and important outcomes? Were benefits and costs that went beyond 1 year discounted (3-5\%) and justification given for the discount rate?"10 


\section{CONCLUSIONS}

Our review of economic evaluations on genetic testing and interventions found that the studies published between 2004 and 2009 were of fairly high quality with a mean quality score of 89.8 of 100. Our findings suggest that no statistically significant improvement in quality of such evaluations occurred from the period 1995-2004 to 2004-2009. Although the mean quality score was fairly high, assessment of missing quality criteria identified a number of areas for improvement, including discussion of the direction and magnitude of potential biases. In addition, we found aspects of QHES methodology for grading the quality of economic evaluations to be ambiguous, such as the inclusion of multiple distinct issues in one criterion or the use of poorly identified terminology. Additional research is needed to modify, clarify, and optimize the QHES quality grading system.

\section{ACKNOWLEDGMENTS}

This research was supported by a grant from the Ontario Research Fund (RE01-061). KAS is supported by a Canada Research Chair award and the Sherman Family Chair in Genomic Medicine. The Pharmacoeconomics Research Unit is supported by Cancer Care Ontario and the Ministry of Health. This research does not reflect the views of the funders. The authors thank Jean Yong from the Pharmacoeconomics Research Unit at Cancer Care Ontario for her contributions.

\section{REFERENCES}

1. Veenstra DL, Higashi MK, Phillips KA. Assessing the cost-effectiveness of pharmacogenomics. AAPS PharmSci 2000;2:1-11.

2. Hubbart HB. A primer on economic evaluations related to expansion of newborn screening for genetic and metabolic disorders. J Obstet Gynecol Neonatal Nurs 2006;35:692-699.

3. Drummond MF, Sculpher MJ, Torrance GW, O'Brien BJ, Stoddart GL. Methods for the economic evaluation of health care programs, 3rd ed. New York: Oxford University Press, 2005

4. Dervieux T, Bala MV. Overview of the pharmacoeconomics of pharmacogenetics. Pharmacogenomics 2006;7:1175-1184.

5. Carlson JJ, Henrikson NB, Veenstra DL, Ramsey SD. Economic analyses of human genetics services: a systematic review. Genet Med 2005;7:519-523.

6. Griffith GL, Edwards RT, Gray J. Cancer genetics services: a systematic review of the economic evidence and issues. Br J Cancer 2004;90:16971703.

7. Dendukuri N, Khetani K, McIsaac M, Brophy J. Testing for HER2-positive breast cancer: a systematic review and cost-effectiveness analysis. CMAJ 2007;176:1429-1434.

8. Rogowski W. Genetic screening by DNA technology: a systematic review of health economic evidence. Int J Technol Assess Health Care 2006;22:327-337.

9. Vegter S, Boersma C, Rozenbaum M. Wilffert B, Navis G, Postma M. Pharmacoeconomic evaluations of pharmacogenetic and genomic screening programmes: a systematic review on content and adherence to guidelines. Pharmacoeconomics 2008;26:569-587.

10. Chiou CF, Hay JW, Wallace JF, et al. Development and validation of a grading system for the quality of cost-effectiveness studies. Med Care 2003;41:32-44.

11. Musci T, Caughey A. Cost-effectiveness analysis of prenatal populationbased fragile X carrier screening. Am J Obstet Gynecol 2005;192:19051915.

12. Meckley I, Veenstra D. Screening for the alpha-adducin Gly460Trp variant in hypertensive patients: a cost-effectiveness analysis. Pharmacogenet Genomics 2006;16:139-147.

13. Costa-Scharplatz M, van Asselt AD, Bachmann LM, Kessels AG, Severens JL. Cost-effectiveness of pharmacogenetic testing to predict treatment response to angiotensin-converting enzyme inhibitor. Pharmacogenet Genomics 2007; 17:359-368.

14. Veenstra DL, Harris J, Gibson RL, Rosenfeld M, Burke W, Watts C. Pharmacogenomic testing to prevent aminoglycoside-induced hearing loss in cystic fibrosis patients: potential impact on clinical, patient, and economic outcomes. Genet Med 2007;9:695-704.
15. Warren E, Anderson R, Proos AL, Burnett LB, Barlow-Stewart K, Hall J. Cost-effectiveness of a school-based Tay-Sachs and cystic fibrosis genetic carrier screening program. Genet Med 2005;7:484-494.

16. Wei S, Quigg MH, Monaghan KG. Is cystic fibrosis carrier screening cost effective? Community Genet 2007;10:103-109.

17. Gagné G, Reinharz D, Laflamme N, Adam PC, Rousseau F. Hereditary hemochromatosis screening: effect of mutation penetrance and prevalence on cost-effectiveness of testing algorithms. Clin Genet 2007;71:46-58.

18. Cooper $\mathrm{K}$, Bryant $\mathrm{J}$, Picot $\mathrm{J}$, et al. A decision analysis model for diagnostic strategies using DNA testing for hereditary haemochromatosis in at risk populations. QJM 2008;101:631-641.

19. Winter J, Walker A, Shapiro D, Gaffney D, Spooner RJ, Mills PR. Costeffectiveness of thiopurine methyltransferase genotype screening in patients about to commence azathioprine therapy for treatment of inflammatory bowel disease. Aliment Pharmacol Ther 2004;20:593-599.

20. Dubinsky MC, Reyes E, Ofman J, Chiou C, Wade S, Sandborn WJ. A cost-effectiveness analysis of alternative disease management strategies in patients with Crohn's disease treated with azathioprine or 6-mercaptopurine. Am J Gastroenterol 2005;100:2239-2247.

21. Priest VL, Begg EJ, Gardiner SJ, et al. Pharmacoeconomic analyses of azathioprine, methotrexate and prospective pharmacogenetic testing for the management of inflammatory bowel disease. Pharmacoeconomics 2006;24: 767-781.

22. Welton NJ, Johnstone EC, David SP, Munafo MR. A cost-effectiveness analysis of genetic testing of the DRD2 Taq1A polymorphism to aid treatment choice for smoking cessation. Nicotine Tob Res 2008;10:231-240.

23. Heitjan DF, Asch DA, Ray R, Rukstalis M, Patterson F, Lerman C. Costeffectiveness of pharmacogenetic testing to tailor smoking-cessation treatment. Pharmacogenomics J 2008;8:391-399.

24. Balmana J, Sanz J, Bonfill X, et al. Genetic counseling program in familial breast cancer: analysis of its effectiveness, cost and cost-effectiveness ratio. Int $J$ Cancer 2004;112:647-652.

25. Phillips KA, Ackerman MJ, Sakowski J, Berul CI. Cost-effectiveness analysis of genetic testing for familial long QT syndrome in symptomatic index cases. Heart Rhythm 2005;2:1294-1300.

26. Kievit W, de Bruin JH, Adang EM, et al. Cost-effectiveness of a new strategy to identify HNPCC patients. Gut 2005;54:97-102

27. Perlis RH, Ganz DA, Avorn J, et al. Pharmacogenetic testing in the clinical management of schizophrenia: a decision-analytic model. J Clin Psychopharmacol 2005;25:427-434.

28. Breheny N, Geelhoed E, Goldblatt J, Ee H, O'Leary P. Economic evaluation of the familial cancer programme in Western Australia: predictive genetic testing for familial adenomatous polyposis and hereditary non-polyposis colorectal carcinoma. Community Genet 2006:9;98-106.

29. Morelle M, Hasle E, Treilleux I, et al. Cost-effectiveness analysis of strategies for HER2 testing of breast cancer patients in France. Int J Technol Assess Health Care 2006;22:396-401.

30. Olsen KR, Bojesen SE, Gerdes AM, Lindorff-Larsen K, Bernstein IT Cost-effectiveness of surveillance programs for families at high and moderate risk of hereditary non-polyposis colorectal cancer International. Int $J$ Technol Assess Health Care 2007;23:89-95

31. Nielsen M, Hes FJ, Vasen HF, van den Hout W. Cost-utility analysis of genetic screening in families of patients with germline MUTYH mutations. BMC Med Genet 2007;8:1-10.

32. Mersereau JE, Plunkett BA, Cedars MI. Preimplantation genetic screening in older women: a cost-effectiveness analysis. Fertil Steril 2008;90:592-598.

33. Eckman MH, Rosand J, Greenberg SM, Gage BF. Cost-effectiveness of using pharmacogenetic information in warfarin dosing for patients with nonvalvular atrial fibrillation. Ann Intern Med 2009;150:73-83.

34. Hollingsworth B, Harris A. Economic evaluation of prenatal population screening for fragile X syndrome. Community Genet 2005;8:68-72.

35. Newman WG, Hamilton S, Ayres J, et al. Array comparative genomic hybridization for diagnosis of developmental delay: an exploratory costconsequences analysis. Clin Genet 2007;71:254-259.

36. Rogowski WH. The cost-effectiveness of screening for hereditary hemochromatosis in Germany: a remodeling study. Med Decis Making 2009;29: 224-238.

37. Canadian Agency for Drugs and Technologies in Health. Guidelines for the economic evaluation of health technologies. Ottawa, Canada: Canadian Agency for Drugs and Technologies in Health, 2006.

38. College Voor Zorgverzekeringen. Guidelines for pharmacoeconomic research, updated version. Diemen, Netherlands: College Voor Zorgverzekeringen, 2006.

39. The Pharmaceutical Benefits Board. General guidelines for economic evaluations from the Pharmaceutical Benefits Board. Sweden: The Pharmaceutical Benefits Board, 2003 